\title{
Luciscit hoc jam : l'Heautontimoroumenos et le temps nocturne dans la dramaturgie classique
}

\section{Hélène Baby}

\section{(2) OpenEdition}

1 Journals

Édition électronique

URL : http://journals.openedition.org/rhetorique/567

DOI : 10.4000/rhetorique.567

ISSN : 2270-6909

Éditeur

UGA Éditions/Université Grenoble Alpes

Édition imprimée

ISBN : 978-2-37747-029-7

\section{Référence électronique}

Hélène Baby, «Luciscit hoc jam : l'Heautontimoroumenos et le temps nocturne dans la dramaturgie classique ", Exercices de rhétorique [En ligne], 10 | 2017, mis en ligne le 20 décembre 2017, consulté le 02 octobre 2020. URL : http://journals.openedition.org/rhetorique/567 ; DOI : https://doi.org/10.4000/ rhetorique.567

Ce document a été généré automatiquement le 2 octobre 2020.

\section{(c) (7) ()}

Les contenus de la revue Exercices de rhétorique sont mis à disposition selon les termes de la Licence Creative Commons Attribution - Pas d'Utilisation Commerciale - Partage dans les Mêmes Conditions 4.0 International. 


\title{
Luciscit hoc jam : l' Heautontimoroumenos et le temps nocturne dans la dramaturgie classique
}

\author{
Hélène Baby
}

1 En 1764, Diderot dans ses Réflexions sur Térence écrivait : "Quel est l'homme de lettres qui n'ait pas lu plus d'une fois son Térence, et qui ne le sache presque par cœur?». Commenté depuis l'Antiquité, édité en Europe des centaines de fois entre 1470 et $1600^{1}$, le poète latin est loué non seulement pour la qualité de son elocutio, mais aussi pour les vertus morales de ses comédies. Au XVII ${ }^{\mathrm{e}}$ siècle, lors de la théorisation des règles classiques, il passe pour le modèle des poètes comiques, et se trouve ainsi au cœur d'une très longue querelle, celle qui oppose l'abbé d'Aubignac et Gilles Ménage sur la durée de l'intrigue de l'Heautontimoroumenos. Hédelin affirmait qu'elle était inférieure à dix heures, et dans tous les cas à douze heures, ce que contestait son adversaire.

2 Revenir aujourd'hui sur cette querelle ne consiste pas à déterminer lequel des deux avait raison, mais bien à en saisir les enjeux profonds : au-delà du minutage de la nuit térentienne, cette dispute savante souligne la difficulté à théoriser l'action fictive, et en particulier les événements censés se passer la nuit. La comédie de Térence permet, grâce à son exceptionnelle organisation en deux périodes temporelles séparées par une nuit, de poser la question du temps nocturne dans la dramaturgie classique et en particulier son rapport à l'unité de temps.

\section{Quelques préalables}

\subsection{Les entractes et d'Aubignac : un temps véritable}

3 À l'âge classique, il est désormais entendu, pour reprendre la belle métaphore de l'ombre portée de Durval ${ }^{2}$, que la proportion doit être raisonnable entre l'ombre et le 
sujet, qu'il s'agisse de douze, de vingt-quatre, voire de trente heures sous la plume de Corneille. Pour permettre cette proportion légèrement agrandie du temps fictif de l'intrigue par rapport au temps réel de la représentation, dramaturges et théoriciens du poème dramatique s'accordent sur la fabrique d'une structure discontinue où, entre les cinq actes qui calquent chacun une durée réelle grâce à la liaison des scènes, les quatre entractes fournissent les intervalles de temps nécessaires à l'accomplissement de l'action fictive.

On sait bien ainsi que l'intervalle pourvoyeur de la discontinuité scénique est au cœur de la dramaturgie classique: on sait moins que c'est aussi la conviction de l'abbé d'Aubignac, que la critique lit parfois un peu vite en affirmant que l'auteur de $L a$ Pratique du théâtre serait favorable à la suppression des intervalles ${ }^{3}$, alors même que le chapitre vi du livre III préconise clairement leur maintien, à la fois pour permettre aux spectateurs de souffler et de détendre leur attention, mais aussi pour placer hors-scène des actions accomplies par les personnages de la fiction ${ }^{4}$. D'ailleurs, c'est aussi à propos de Térence que d'Aubignac prend position en faveur des intervalles, en s'opposant à l'hypothèse de Donat sur la " continuation » des actes dans L'Eunuque, suggestion que le théoricien taxe de « confusion imaginaire » :

[...] mais dans les Actes, il n'y a pas le moindre soupçon de cette confusion imaginaire dont parle Donat, et souvent les Latins ont été si soigneux d'en marquer la distinction ${ }^{5}[. .$.$] .$

D'Aubignac est convaincu de la nécessité de la discontinuité dans la représentation, mais à la condition que cette discontinuité réelle recouvre néanmoins une continuité dans la fiction. Conception que l'on peut qualifier d'extrême, voire d'extrémiste, car elle repose sur l'idée que le temps fictif est un temps réel : «le jour représenté dans lequel on suppose que l'Action du Théâtre est arrivée, doit être pris pour un temps véritable à l'égard de cette action ${ }^{6} »$. Ce que Pierre Pasquier signale comme une position « originale, sinon marginale », comportant des « risques de l'objectivation » et entraînant les errements du théoricien qui se voit contraint au minutage dudit temps fictif ${ }^{7}$.

5 Il apparaît que la querelle de l'Heautontimoroumenos permet de prolonger cette réflexion et d'apporter des précisions sur la position de l'abbé d'Aubignac, qui n'est peut-être pas si marginale qu'elle le semble et qui, au fond, propose une nouvelle hiérarchie théorique entre longueur et continuité, subordonnant la première à la seconde.

\subsection{La querelle : description}

6 L'Heautontimoroumenos, qu'on appelle aussi la "troisième comédie de Térence " pour la place qu'elle occupe dans les éditions de son théâtre, met en scène les amours de deux jeunes gens, Clinia et Clitiphon, aux prises avec leurs pères respectifs, Ménédème et Chrémès, et se termine, après une reconnaissance, par un double mariage. L'action commence à la fin d'une journée et se termine le lendemain, une nuit séparant le vers $409 \mathrm{du}$ vers 410 , comme l'indique Chrémès au début de l'acte III déclarant: " Luciscit hoc jam », expression signalant le lever du jour, en écho au verbe "vesperascit » prononcé par Syrus à l'acte II, et confirmée par les plaintes de Chrémès sur la débauche nocturne qui s'est faite à ses dépens ${ }^{8}$.

7 Le problème posé par cette interruption nocturne est bien connu, et commenté par tous les érudits, lecteurs et éditeurs de Térence, qui comme Muret $^{9}$, Gouveia ${ }^{10}$, Scaliger 
ou Eugraphius, consacrent une glose au vers 410 et à l'expression Luciscit hoc jam. Si d'Aubignac et Ménage, suivant en ceci leurs prédécesseurs, lecteurs, éditeurs et commentateurs de Térence, s'accordent sans difficulté sur le fait que l'action occupe bien une soirée et un matin, ils s'opposent quant à la durée de la nuit, en une querelle dont les étapes éditoriales sont elles aussi bien connues ${ }^{11}$, et qui dura plus de trente ans. Et, en dépit de la mort de son adversaire en 1676, Ménage saisit l'occasion que lui fournit l'édition des comédies de Térence par la célèbre helléniste Anne Dacier ${ }^{12}$ pour publier une troisième fois ses arguments en 1690, dans un ouvrage intitulé Discours de Ménage sur l'Heautontimoroumenos de Térence ${ }^{13}$. Même si l'accumulation des justifications dans son Avertissement n'atténue guère l'indécence du procédé qui consiste à polémiquer avec un mort, cette publication tardive revêt néanmoins tout l'intérêt de présenter un sommaire très commode : Ménage y résume cinquante années de discussion et, en dépit d'un point de vue évidemment partial, il souligne ainsi les points saillants de la dispute.

Ceux-ci sont globalement de deux ordres, anthropologiques et linguistiques, les uns portant sur la civilisation romaine et les autres sur les temps verbaux et les erreurs de traduction. Puisque l'action de la comédie se passe le jour des Dionysies («Dionysia hic sunt hodie ", dit Chrémès dès la première scène), d'Aubignac et Ménage vont croiser le fer sur la date hypothétique de cette fête, car tout le monde sait bien que les nuits sont courtes l'été mais longues l'hiver... Ainsi d'Aubignac, estimant qu'il s'agit de la fête de Pithégie, premier jour des Anthestéries qu'il situe pendant le mois d'avril, assure qu'il s'agit d'une nuit de printemps ; de son côté, Ménage, estimant que le mois Anthestérion est à cheval entre janvier et février, compte sur une nuit bien plus longue. Autre argument " ethnologique », celui qui se développe à partir du bain que prend Antiphila le deuxième jour ("si jam laverit ", acte III, scène 1) : la dispute se concentre sur l'heure des ablutions des Romaines, d'Aubignac affirmant que c'était très tôt, et Ménage étant partisan d'un horaire plus tardif. «Il est vrai néanmoins qu'on se baignait le matin : mais non pas deux heures avant le Soleil », écrit Ménage ${ }^{14}$.

9 Moins folkloriques apparaissent les débats linguistiques, parmi lesquels nous retiendrons la dispute portant sur les verbes labora, luciscit et vesperascit. Soucieux de raccourcir la nuit, l'abbé d'Aubignac estime que Ménédème a déjà quitté son champ et que le travail que le texte lui impute (ne labora) n'est que le fardeau des pesants râteaux qu'il ramène une fois sa journée finie. De même il traduit «Luciscit hoc jam » par « il commence à faire jour », et " vesperascit » par « il fait nuit noire » et justifie longuement dans son Térence Justifié ${ }^{15}$ cette incohérence grammaticale que Ménage ne se prive pas de lui faire remarquer ${ }^{16}$, puisque l'abbé se contredit en affectant tantôt un sens inchoatif, tantôt un sens accompli à la même forme verbale. Ménage, quant à lui, traduit « vesperascit » par « le soleil se couche ${ }^{17}$ » et « luciscit » par « le jour commence ».

\subsection{La postérité au piège de l'érudition}

Cette disputatio qui déclenche un emballement érudit se voit très sévèrement jugée par la postérité. Antoine Adam écrit : «Le sujet peut en paraître à la fois pédantesque et frivole ${ }^{18}$ ». Maurice Descotes renchérit : "à cette date, se battre sur les règles est un exercice gratuit: leur cause est désormais gagnée. La contestation ne peut donc conduire qu'à raffiner, à byzantiniser [...] des deux côtés, on s'applique à "minuter" la pièce ${ }^{19}$ "; « le débat ne roule que sur des subtilités ${ }^{20}$ » écrit à son tour Elvire Samfiresco. Ces jugements unanimement négatifs découlent de ce que la querelle semble se réduire 
à la seule question de l'unité de temps, question dont précisément le principe est réglé au moment de cet affrontement savant, comme l'écrit Maurice Descotes : ni Ménage, ni d'Aubignac, ni Chapelain, ni Corneille ne contestent plus la nécessité de la régularité, en d'autres termes la proportion raisonnable (de douze ou de vingt-quatre heures) entre la chose imitée et la chose qui imite, et chacun a désormais tiré les conclusions du raisonnement de Castelvetro.

Pas plus qu'il n'est possible de faire croire au public que plusieurs jours et plusieurs nuits se sont écoulés quand il sait pertinemment que quelques heures seulement se sont passées : une telle illusion est impossible puisque, en tout état de cause, elle doit se fonder sur les sens ${ }^{21}$.

Aussi, le débat qui oppose d'Aubignac et Ménage, réduit à cette seule question de l'unité de temps, semble justement stérile : puisqu'il y a consensus sur le principe fondateur de l'intervalle comme pourvoyeur de temps et d'action, il n'est au fond question que d'une différence de degré, et pas de nature, ce qui revient en effet à «byzantiniser »... D'autant que ladite différence de degré est finalement bien faible, ne variant, de d'Aubignac à Ménage, que de quelques heures quant à l'extension maximale que le spectateur serait susceptible de conférer à la durée de l'action fictive pendant l'intervalle nocturne.

11 Est-ce si simple ? On peut penser que la postérité critique a été prise au piège de la folle érudition des deux débatteurs, et de celle de Ménage en particulier, qui a construit son argumentation uniquement sur le minutage de la nuit. Alors que d'Aubignac, présentant les enjeux de la dispute, écrit clairement qu'il y «deux difficultés qu'[il] s'efforcera de résoudre en ce discours" - "la règle des douze heures » et le «temps perdu $^{22}$ »-, Ménage, lui, ne retient que la première: "J'ai à prouver que l' Heautontimoroumenos de Térence comprend plus de douze heures ${ }^{23}$ ». Il fait ainsi disparaître le deuxième pilier de la dispute, celui qui porte sur le "vide", et dont découle pourtant la durée de douze heures. Et c'est in extremis qu'il condescend à se pencher sur ce deuxième point : on voit bien que, sur les 264 pages de son Discours, il en consacre 242 à la question des dix ou douze heures, et que, à partir de la page 242 , il expédie en moins d'une vingtaine de pages une dernière question, qui est sans doute la «vraie» question, celle de la discontinuité. De surcroît, c'est seulement dans la dernière version de cet ouvrage qu'il accepte de lui consacrer un chapitre à part entière, en en faisant la seconde "partie » de son Discours. Ses précédentes réponses, elles, n'étaient pas divisées en deux points.

12 Si Ménage ne s'appesantit pas, c'est qu'il entend sans doute éviter de souligner son accord avec ce point théorique central de la théorie aubignacienne, qui est de proscrire le «temps perdu». En effet, sous l'avalanche érudite portant sur l'aspect inchoatif des verbes, le sens de vesper, les ablutions, les mœurs de la campagne, la durée des nuits d'hiver et le calendrier des fêtes romaines, vont émerger un point d'ancrage et un point d'accord : quelle que soit la durée de la nuit térentienne, les deux savants finissent par s'entendre, particulièrement sur la nécessité de personnages qui ne dorment pas et font avancer l'action fictive pendant l'intervalle nocturne.

13 C'est pourquoi la querelle de Ménage et de d'Aubignac sur la durée de la nuit térentienne n'est peut-être pas frivole, pédantesque ou futile : elle traduit la façon de considérer l'action fictive et permet d'établir les critères de son éventuelle continuité. 


\title{
2. De l'intervalle et du sommeil : qui « se mettrait au $\operatorname{lit}^{24}$ »?
}

\subsection{Scaliger, d'Aubignac, Mme Dacier et le « troisième intervalle »}

La question du sommeil n'était-elle pas déjà celle que posait la fameuse glose de Scaliger sur le vers 410 de l'Heautontimoroumenos? On sait que l'auteur des Poetices libri septem consacre une réflexion originale à la comédie de Térence :

\begin{abstract}
vasta, inquiunt, et hians, atque inanis comoedia est. Tota namque intercedit nox. Nam per initia cenam curant. Postea Chremes ait: Lucescit. Sane igitur abiit nox. Haec est illorum obiectio, quam sic diluimus : datam actamque fabulam ludis Megalensibus. Itaque dimidium fabulae actum vesperi, noctem transactam ludis, alterum dimidium reliquum sub lucem. Unam igitur quasi duas. Id quod cum esset per praeconem pronuntiatum iussu aedilium, pollicitus est ipse quoque poeta in prologo versu illo: duplex quae ex argumento facta est simplici $i^{25}$.

C'est, disent-ils, une comédie tout à la fois mal dégrossie, mal enchaînée et sans intérêt ; et de fait une nuit entière est intercalée. En effet, au début, on s'occupe du dîner, ensuite Chrémès dit « le jour se lève ». C'est donc évidemment que la nuit est finie. C'est là leur reproche que nous dissipons en disant que la pièce a été donnée et jouée aux jeux mégalésiens ; qu'en conséquence la moitié de la pièce a été jouée le soir, que la nuit s'est passée aux jeux ; que la seconde moitié restante a été jouée au point du jour ; donc qu'une seule pièce en fait pour ainsi dire deux, - ce qui avait été annoncé par le héraut sur l'ordre des édiles - ce qu'a promis également le poète lui-même dans le prologue par ce vers : "comédie double qui a été composée à partir d'un seul sujet ».
\end{abstract}

La solution proposée est pour le moins déconcertante : Scaliger rend compte de la nuit entre les actes II et III (entre les vers 409 et 410) en rappelant que la comédie de Térence a été représentée pendant les jeux mégalésiens, dont les festivités duraient toute la nuit, et qu'elle a été représentée en deux temps, les deux premiers actes un soir, et les trois derniers le lendemain. Cette hypothèse lui permet d'ailleurs d'expliciter la phrase du prologue, «duplex quae ex argumento facta est simplici ». Cette glose a gêné des générations de critiques, depuis d'Aubignac et Ménage jusqu'à Harold W. Lawton qui a consacré quelques pages à ce surprenant passage dont il ne parvient pas à justifier la pertinence, comme le montre son article qui se conclut ainsi :

Enfin il est amusant de trouver chez l'érudit formidable qu'était Scaliger soit une faiblesse, soit l'élan d'une imagination qu'on n'aurait jamais pensé trouver chez lui ${ }^{26}$.

Faiblesse de Scaliger ? En tout cas, faiblesse reprise par Madame Dacier dans son édition des comédies de Térence, où, dans ses Remarques sur l'Heautontimoroumenos, elle reprend cette hypothèse :

On ne peut pas douter que cette comédie n'ait été jouée à deux reprises ; les deux premiers actes furent joués le soir après le coucher du soleil, et les trois autres le matin à la pointe du jour ${ }^{27}$.

Sans se contenter de répéter ce qu'avait dit Scaliger, Mme Dacier, afin de prouver le caractère non exceptionnel de cette pratique ${ }^{28}$, renchérit en ajoutant l'exemple du Plutus d'Aristophane et insiste sur le fait que le temps nocturne comporte une action liée au sujet :

Mais ce qui mérite d'être bien remarqué ici, c'est que ce troisième intervalle entre

dans l'action, et devient une partie du sujet ${ }^{29}$.

Ce qu'elle appelle curieusement le "troisième intervalle » suppose l'existence d'un espace-temps partagé, ni propre aux seuls personnages, ni propre aux seuls 
spectateurs : certains dorment, certains veillent, en d'autres termes tous font quelque chose, et au même rythme. Comment ne pas penser à l'invention de Beaumarchais, certes aporétique mais parlante, avec ses « jeux d'entracte » inventés dans son Eugénie? Ils correspondent, dans le principe, à ce troisième intervalle proposé par Mme Dacier, à mi-chemin entre les personnages et les spectateurs.

L'action théâtrale ne reposant jamais, j'ai pensé qu'on pourrait essayer de lier un acte à celui qui le suit par une action pantomime qui soutiendrait sans la fatiguer l'attention des spectateurs, et indiquerait ce qui se passe derrière la scène pendant l'entracte. Je l'ai désignée entre chaque acte. Tout ce qui tend à donner de la vérité est précieux dans le drame sérieux, et l'illusion tient plus aux petites choses qu'aux grandes ${ }^{30}$.

À bien lire, la solution préconisée par Scaliger portait en gestation la théorie radicale de d'Aubignac, car elle justifiait la nuit fictive par la nuit réelle que vivaient les spectateurs. Voilà pourquoi l'auteur du Térence justifié, qui a bien compris ce point, défend l'érudit italien en affirmant qu'il «fait grâce à Térence » : il accepte le principe de l'hypothèse de Scaliger, même si elle le gêne, car elle suppose que chaque représentation de l'Heautontimoroumenos doive maintenir éveillés les spectateurs, ce qui n'est possible que lors de la fameuse fête des Anthestéries.

[...] la rencontre de la représentation ne peut excuser le travail du poète, ou bien

l'on ne pourrait jamais la représenter qu'aux jeux Mégaliens ${ }^{31}$.

C'est le fait que cette coïncidence ne serait possible qu'une fois par an qui pose problème, mais pas le principe de cette coïncidence, comme le dit bien Mme Dacier : l'idée scaligérienne selon laquelle personne ne dort, pas plus les personnages que les spectateurs, correspond à la théorisation du temps que proposera d'Aubignac.

\subsection{Dormir ou ne pas dormir ? Où Ménage rejoint d'Aubignac}

16 Que faire pendant la nuit? Que faire des intervalles? Dormir ou ne pas dormir? telle est la question que pose finalement la querelle de l'Heautontimoroumenos. Ce n'est en effet pas un hasard si Ménage clôt son Discours sur le problème de savoir qui se couche et qui dort. Alors que d'Aubignac a affirmé que la maisonnée de Chrémès n'a pas pris de repos après le souper :

[...] le Poète, pour prévenir cela, fait dire à Chrémès que les esclaves parlaient à l'oreille avec leurs maîtres lorsqu'il est sorti [...] D'où nous apprenons qu'ils ne sont pas demeurés oisifs après le souper [...] et qu'ils n'étaient ni couchés ni endormis, mais plutôt qu'ils disposaient les intrigues des Actes suivants ${ }^{32}[\ldots]$.

et qu'il a même précisé que les Romains avaient prévu des «lampes qui dur[aient] longtemps ${ }^{33}$ ", Ménage, s'obstinant à s'opposer à lui, argumente habilement, en contredisant son adversaire tantôt sur la différence entre le repos et le sommeil, et tantôt sur le nombre de personnages endormis. Ainsi il rétorque que Chrémès s'est couché mais que se coucher ne signifie pas dormir :

Pour n'avoir point dormi, ce n'est pas à dire qu'on ne se soit point couchés.

De même, contre l'avis de d'Aubignac, il affirme que « Ménédème, Chrémès, Antiphile, Sostrate et la nourrice se so[ie]nt couchés ${ }^{35} »$. Pourtant, il ne va pas jusqu'au bout de cette logique, qui supposerait un sommeil de quelques heures pour tous les personnages, et il admet qu'un groupe de personnages a veillé toute la nuit:

[...] il n'y a point de vide dans l'Heautontimoroumenos : les deux Amants, Clitiphon et

Clinia ; la Courtisane Bacchis, Phrygia, et ses autres Servantes, et les deux Valets, ayant veillé, et ayant toujours fait quelque chose de nécessaire à l'intrigue de la Pièce ${ }^{36}$. 
Ce sont les toutes dernières lignes de son Discours : l'accord est total avec la position de d'Aubignac, quant à la qualité des actions nécessaires pour assurer la continuité de l'action, et, parmi elles, la nécessité de la veille contre le sommeil. Rester éveillé, pour au moins un des personnages, est la condition sine qua non de l'unité de temps, et l'on entend déjà le persiflage de Lessing résumant le postulat classique :

À l'unité de jour, ils ont substitué l'unité de durée; et ils ont admis que l'on compterait pour un seul jour un certain temps, pendant lequel il ne serait question ni de lever ni de coucher du soleil, où personne ne se mettrait au lit, au moins plus d'une fois, quelle que fût d'ailleurs la multitude et la variété des événements qui pourraient s'y passer [...],

écrira-t-il en 1767 à propos de l'usage des règles chez les Français ${ }^{37}$. S'accordant sur le déroulement d'une action "nécessaire " pendant l'intervalle, Ménage et d'Aubignac précisaient ainsi la nature du «mouvement » que Diderot imaginera derrière la scène dans son Discours sur la poésie dramatique (1758):

[...] mais puisque l'action ne s'arrête point, il faut que lorsque le mouvement cesse sur scène, il continue derrière ${ }^{38}$.

Pour reprendre la terminologie du philosophe, la question est bien de savoir de quoi est constitué ce "mouvement » et pas seulement combien de temps il dure. Il s'agit non seulement de considérer un temps "véritable " mais aussi de définir une catégorie d'action qui puisse être continue. Dans cette perspective, le temps "véritable » du sommeil est difficilement recevable, non pas à cause de sa durée mais bien à cause de la nature interruptive de cette action particulière qu'est le sommeil. Car s'il y a "mouvement ", il n'y a pas repos, comme l'explicite Diderot dans la suite de ce même passage :

[...] mais puisque l'action ne s'arrête point, il faut que lorsque le mouvement cesse sur scène, il continue derrière. Point de repos, point de suspension. Si les personnages reparaissaient, et que l'action ne fût pas plus avancée que quand ils ont disparu, ils se seraient tous reposés ou ils auraient été distraits par des occupations étrangères; deux suppositions contraires, sinon à la vérité, du-moins à l'intérêt.

Où l'on retrouve l'hypothèse que d'Aubignac développe au début de son Térence justifié :

[...] vous laissez vos acteurs un fort long temps sans rien faire de nécessaire à ce que vous représentez; ce qui divise l'action du théâtre et y comprend mille autres petites actions dont on n'a pas besoin ${ }^{39}$.

Et, parmi ces «mille petites actions ", ou ces « occupations étrangères », il y a bien sûr le sommeil...

17 À ce point du raisonnement, on voit que la querelle sur la nuit térentienne permet deux enseignements. Le premier, que la nature de l'action fictive pendant les intervalles est aussi importante que sa durée. Le second, que toute la postérité critique a au fond adopté l'attitude de Ménage, et s'est concentrée sur la longueur et pas sur le repos : ce qui explique que les études dramaturgiques ont toujours, avec un bel ensemble, négligé la part réservée à la nuit.

\section{La nuit, marqueur du temps ou marqueur du genre?}

\subsection{La nuit, outil critique}

Depuis que l'analyse dramaturgique existe, nous faisons tous comme si la nuit allait de soi, tant elle est devenue un outil bien pratique pour parier sur l'unité de temps. Ainsi, si l'on regarde de près les remarques faites sur la dramaturgie dans les résumés 
synoptiques des pièces, comme ceux qu'on trouve dans la gigantesque histoire de H.C. Lancaster ${ }^{40}$ ou dans le Dictionnaire de Marc Vuillermoz ${ }^{41}$, on constate un procédé tout à fois récurrent et étonnant: les critiques affirment que telle ou telle pièce "peut» ou «ne peut pas tenir ${ }^{42}$ » en vingt-quatre heures, ou qu'elle «tient en vingtquatre heures ou environ ${ }^{43}$ ». Ainsi Lancaster écrit-il volontiers, et très vaguement, que telle pièce respecte l'unité de temps, avec l'expression " the unity of time is respected ${ }^{44}$ ». Sur La Mort des Enfants d'Hérode de La Calprenède, il affirme : " the unities of time and place are observed ${ }^{45}$ »; ou encore, à propos de L'Innocent malheureux de Grenaille, que «l'action n'excède pas vingt-quatre heures » («the time of the action is not over twenty-four hours ${ }^{46}$ $»)$.

Il serait temps de s'étonner de cette notation de vingt-quatre heures, qui semble aller de soi alors que rien ne la justifie : car, pour « tenir », ou être « environ » dans les vingtquatre heures, encore faut-il qu'il y ait une nuit. Et là, les choses se compliquent car la notation de la nuit est presque toujours absente de ces commentaires: trois fois sur quatre, force est au lecteur d'aller voir dans le détail pourquoi la pièce est dite « dans les vingt-quatre heures ». En d'autres termes, il faut s'interroger sur la facilité avec laquelle la critique dramaturgique affirme « dans les vingt-quatre heures » alors même que l'action ne comporte pas explicitement de nuit. En effet, de deux choses l'une : soit il y a une nuit, soit il n'y en a pas. Si oui, il faudrait le préciser et le prouver; et si non, ce n'est pas vingt-quatre heures mais bien douze heures qu'il faudrait indiquer.

Il semble bien que cet outil critique imaginaire, en l'occurrence la mesure de vingtquatre heures, pousse le lecteur à projeter cette durée sur la pièce et à penser que son intrigue "peut » se passer en vingt-quatre heures parce qu'elle contient une nuit. En somme, dans le meilleur des cas, la description se contente de sous-entendre une temporalité nocturne et, dans le pire des cas, elle l'affecte au petit bonheur à tel ou tel entracte. Un exemple parlant est celui du Comte d'Essex, tragédie de La Calprenède, que Lancaster commente ainsi : «La pièce est composée selon le système classique : vingtquatre heures ${ }^{47}$ ", tandis que le Dictionnaire indique pour la même pièce " environ vingtquatre heures séparées par une nuit ${ }^{48}$ » et place cette nuit à l'entracte entre l'acte III et l'acte IV, sans aucune justification. La seule indication textuelle, elle-même très discutable, est celle de Mme Cécile à la toute fin de l'acte II promettant au comte d'Essex «Et demain au plus tard je viendrai vous revoir ", et tenant parole à l'acte IV. Que peut-on en déduire? Peut-être la nuit se situe-t-elle entre le deuxième et le troisième acte, et non entre le troisième et le quatrième ! Ou peut-être Mme Cécile estelle revenue le voir dans la même journée, sans attendre le lendemain...

21 Autre illustration des habitudes de lecture, celle que fournit l'analyse de Camma de Thomas Corneille. Là où Lancaster écrit "rapide, l'action se déroule en quelques heures $^{49}$ ", le Dictionnaire indique qu'une nuit se passe entre l'acte IV et l'acte $V$ et que l'action dure «environ vingt-quatre heures ${ }^{50}$ ». Exemplaire enfin est le cas de L'Amour tyrannique de Scudéry, dont on sait que Sarasin voulait faire le modèle de la tragédie. Le Dictionnaire précise :

Vraisemblablement, l'action ne déborde pas le cadre des vingt-quatre heures. Selon Sarasin, Scudéry a d'ailleurs pu avoir « quelques heures de reste ». La durée totale de l'action devrait même se ramener à douze heures, si l'on en croit S. Wilma Deierkauf-Holsboer ${ }^{51}$.

Que signifient ce flou et ces contradictions sinon que la nuit ne fait pas partie de la fabrique de l'œuvre, mais de sa réception? C'est bien une «imagination" pour reprendre le mot de l'abbé d'Aubignac: «la règle de vingt-quatre heures, dont on fait 
tant de bruit maintenant, est une imagination de quelques-uns qui ont trompé les autres ${ }^{52} »$.

En fait, l'analyse dramaturgique traite de l'unité de temps comme une mesure de longueur possible au lieu de s'intéresser à ce qui est au cœur de la dramaturgie régulière, à savoir "l'unité de durée ", pour paraphraser Lessing. Quand la pièce est si chargée en événements qu'elle en devient trop dense, une nuit semble nécessaire au lecteur pour rendre acceptable cet entassement événementiel: mais si la nuit est commode à la critique dramatique pour décrire l'unité de temps, elle l'est beaucoup moins pour le dramaturge qui doit assurer la continuité de l'action. C'est pourquoi on constatera facilement que très peu de dramaturges utilisent la nuit pour " gagner » du temps. Et faut-il rappeler que ni Corneille ni Racine n'utilisent la nuit dans leurs tragédies?

\subsection{Que fait-on la nuit?}

Les deux grands auteurs tragiques français, convaincus de la nécessité de respecter l'unité de temps - c'est-à-dire une proportion raisonnable entre réel et fiction n'exploitent en effet jamais la nuit pour " gagner » du temps et faire tenir l'action en vingt-quatre heures. On sait bien que Corneille regrette d'avoir fait expliquer par Don Diègue que Rodrigue avait « pris haleine » en narrant ses exploits nocturnes ${ }^{53}$.

Je me suis toujours repenti d'avoir fait dire au Roi, dans Le Cid, qu'il voulait que Rodrigue se délassât une heure ou deux après la défaite des Maures, avant que de combattre don Sanche. Je l'avais fait pour montrer que la pièce était dans les vingtquatre heures $[. . .]^{54}$.

Mais que déplore exactement Corneille? Ce n'est pas tant d'avoir souligné maladroitement la régularité de la pièce, que d'avoir mis en pleine lumière le repos nécessaire qu'aurait vraisemblablement dû prendre Rodrigue. Que dit-il en effet en réfléchissant sur la nuit mauresque de Rodrigue?

Leur défaite avait assez fatigué Rodrigue toute la nuit pour mériter deux ou trois jours de repos, et même il y avait quelque apparence qu'il n'en était pas échappé sans blessures, quoique je n'en aie rien dit, parce qu'elles n'auraient fait que nuire à la conclusion de l'action ${ }^{55}$.

Ce qui est en jeu ici, ce n'est pas la durée de vingt-quatre heures, de deux, ou de trois jours, c'est le fait qu'il est invraisemblable de livrer un combat (action héroïque digne de la tragédie) sans être blessé, sans récupérer de ses blessures, bref sans se reposer : il n'est pas question de la longueur du temps ici, mais de la nature de l'action. Dès lors, la question que soulève Corneille est la suivante : comment un dramaturge peut-il faire veiller ses personnages sans trop les fatiguer, de façon à ce qu'il soit vraisemblable qu'ils ne se reposent pas? Même d'Aubignac, lors de sa réflexion d'ensemble sur l'étendue de l'action dramatique dans La Pratique, concède que l'action des hommes se passe plutôt le jour que la nuit :

Or nous ne voyons point que régulièrement les hommes agissent devant le Jour, ni qu'ils portent leurs occupations au-delà ; d'où vient que dans tous les États il y a des Magistrats établis pour réprimer ceux qui vaguent la nuit naturellement destinée pour le repos. Et quoiqu'il arrive assez souvent des occasions importantes qui obligent d'agir durant la nuit, cela est extraordinaire ; et quand on veut établir des règles, il les faut toujours prendre sur ce qui se fait le plus communément, et dans l'ordre ${ }^{56}$.

En d'autres termes, agir de nuit demeure exceptionnel. 
, si l'on a besoin de songes ou, comme l'écrit joliment Ménage, si le dramaturge a besoin du "retour des Ombres $^{57}$ ", on relèguera le rêve dans un temps précédant l'action, ou dans un repos momentané: la nuit se trouve alors avantageusement remplacée par la «sieste » ou le « repos » que l'on prend dans la journée. Ainsi, dans L'Innocent malheureux de Grenaille (1639), Hélène, fille de Constantin, dit avoir « cherché du repos » et avoir brièvement rêvé de Minervine («Mon frère, hélas ! étant parti d'ici / J'ai cherché du repos pour charmer mon souci ", acte IV, scène 6). Procédé que l'on retrouve dans La Mort de Sénèque de Tristan L'Hermite (1644) avec l'assoupissement de Sabine qui a rêvé de l'empereur Auguste ("J'étais dans le jardin proche de la fontaine / Et l'agréable cours de ses flots innocents / Avait par son murmure assoupi tous mes sens, / Lorsqu'un songe divin soudain m'a réveillée », III, 2, v. 920-924), moment que le Dictionnaire interprète comme une « sieste $\left.^{58} »\right)$.

On constate aussi que l'utilisation de la nuit dans la tragédie se trouve logiquement circonscrite à une action notoirement nocturne, comme, par exemple, le viol de Lucrèce, les meurtres commis par les Danaïdes sur leurs époux endormis, l'enterrement secret de Polynice (voir Les Danaïdes de Gombauld, l'Antigone de Rotrou, les Lucrèce de Chevreau et de Du Ryer), toutes actions que le théâtre tragique exploite d'ailleurs avant 1650. En effet, si l'on suit la périodisation de Lancaster (1635-1651, puis 1652-1672), on voit que la tragédie s'efforce progressivement de supprimer les sujets impliquant une activité nocturne : alors que, entre 1635 et 1651, la question de la nuit et du repos ne fait guère problème ${ }^{59}$, entre 1652 et 1672 , seules trois tragédies placent une action de nuit, La Mort de Démétrius de Boyer, Pausanias de Quinault, et Ariane de T. Corneille, et, parmi elles, seul Quinault exploite la confusion due à l'obscurité, lors de laquelle Pausanias tue Cléonice pensant affronter son rival. Boyer et T. Corneille n'utilisent la nuit que pour y placer la tentative d'évasion ou la fuite (respectivement d'Alexandre et de Thésée). Il faudrait mener cette enquête de façon exhaustive sur le corpus tragicomique mais on constaterait aisément qu'il y a de moins en moins de nuits à la fois dans la tragédie et dans la tragi-comédie, en d'autres termes dans ce qu'on peut appeler le théâtre sérieux.

En même temps que la nuit déserte le théâtre sérieux, elle persiste et gagne dans la dramaturgie comique. Comme l'a montré l'exploit invraisemblable de Rodrigue, les personnages susceptibles de veiller avec vraisemblance ainsi que les actions susceptibles d'être nocturnes se trouvent plus facilement dans l'humanité moyenne, qui peut se livrer aux beuveries et festins, ou aux enlèvements à la faveur de l'ombre, toutes actions réservées aux domestiques, aux personnages de romans, bref au personnel de la comédie. Aussi la comédie après 1660 prolonge-t-elle volontiers la tradition du quiproquo nocturne qui irrigue la comédie française depuis sa renaissance avec Corneille dans les années 1630, ainsi que celle de la scena di notte sous l'influence de la commedia dell'arte. Et l'on sait bien que les pièces de Molière, et des plus célèbres d'entre elles (Le Dépit amoureux, l'École des Femmes, Georges Dandin, Dom Juan, etc.), utilisent les scènes nocturnes, se plaçant dans l'héritage des malentendus comiques ou tragi-comiques et des scènes grivoises tels qu'en offraient La Place Royale, Les Galanteries du Duc d'Ossonne, Laure persécutée, Dom Japhet d'Arménie ${ }^{60}$ : la nuit, propice aux erreurs, aux lutineries et aux renversements burlesques, devient à l'âge classique l'apanage de la comédie, comme l'indique le titre exemplaire de la comédie de Nanteuil en 1672, Les Brouilleries nocturnes ${ }^{61}$. Peut-être parce que la tragédie développe, à la même époque, un 
autre type d'action, où l'erreur n'est pas due à une fausse apparence mais à l'aveuglement intérieur du personnage.

L'Heautontimoroumenos offre en somme un cas d'école, et la querelle sur cette troisième comédie de Térence a permis de proposer une réflexion essentielle sur la dramaturgie du temps nocturne. La nuit térentienne illustre que la nuit au théâtre n'est pas faite pour le sommeil et, partant, qu'elle convient mieux, par la nature des actions qu'elle engage, aux sujets comiques qu'aux sujets héroïques et sérieux.

Paradoxalement donc, la nuit, loin d'être un outil pour la tragédie classique, est son ennemie, soit parce qu'elle interrompt l'action, soit parce qu'elle en menace la vraisemblance. Ce qu'ont bien compris, et Corneille, et Racine, limitant dès lors leurs actions tragiques dans un temps diurne de douze heures, et donnant finalement raison à la théorie de d'Aubignac.

\section{NOTES}

1. Voir H. W. Lawton, Contribution à l'histoire de l'humanisme : Térence en France au XVI siècle, [1926], réimp. Genève, Slatkine, 1970.

2. "La représentation n'est que l'ombre de l'histoire", Discours à Cliton (1637), dans Temps de préfaces, éd. G. Dotoli, Paris, Klincksieck, 1996, p. 279.

3. P. Pasquier, «L'ombre du temps : réflexion sur le statut du temps dramatique dans le discours esthétique du XVII ${ }^{\mathrm{e}}$ siècle ", Littératures Classiques, ${ }^{\circ} 43$, automne 2001, p. 89-116; la réflexion sur «l'hypothèse de la suppression » de l'intervalle se situe p. 108.

4. Voir La Pratique du théâtre [1657], éd. H. Baby, Paris, Champion, 2011, III, VI « Des Intervalles des Actes », p. $350:$ : [...] l'expérience nous apprend que les hommes n'ont point d'attention assez forte pour supporter une Pièce de Théâtre toute entière et sans [aucun] relâche »; ibid., p. 353 : «Le principal avantage que le Poète peut tirer des Intervalles des Actes est, Que par ce moyen il se peut décharger de toutes les choses embarrassantes, et de toutes les superfluités de son Sujet $»$.

5. Op. cit., III, v, « Des Actes », p. 335.

6. Op. cit., I, VII, « Du mélange de la Représentation avec la Vérité de l'Action théâtrale », p. 87.

7. P. Pasquier, op. cit., p. 103.

8. "J'ai débouché tous mes tonneaux, toutes mes cruches. Elle a mis tout le monde sur les dents, et ce n'est là qu'une seule nuit » (vers 455 sq.).

9. C'est par exemple Marc-Antoine Muret qui rétablit en 1554 l'orthographe « luciscit » au lieu de "lucescit » que l'on trouve encore souvent au XVII (dans Muret, Catullus. Et in eum commentarius M. Antonii Mureti, Venise, Paul Manuce, 1554). Voir M.-A. Muret, Terentius [...] emendatus, Venise, Paul Manuce, 1555, scholie 21: "In omnibus libris, praeter Manutianis, locus hic inepte et scriptus, et interpunctus erat, hoc modo: Lucescit : hoc jam cesso pultare ostium, etcet. Quae sit autem vis hujus orationis, Luciscit hoc jam, dixi quodam loco in commentario in Catullum "; "Dans tous les livres, excepté ceux de Manuce, ce passage avait été écrit et ponctué avec maladresse, de la façon suivante: Lucescit: hoc jam cesso pultare ostium, etcet. Or j'ai déjà dit dans un passage du commentaire sur Catulle que ces mots, Luciscit hoc jam [Voici qu'il commence à faire jour], constituent l'élément fondamental du discours » (trad. M. Roux, Marc-Antoine Muret, lecteur et 
éditeur de Térence, Université Lumière-Lyon 2, 2010, http://www.enssib.fr/bibliothequenumerique/documents/48932-marc-antoine-muret-lecteur-et-editeur-de-terence.pdf).

10. Voir Antonii Goveani Opera Juridica, Philologica, Philosophica [...], Roterodami, Apud Henricum Beman, MDCCLXVI. On lit à la page 597 : «Dormiri in hac comcedia necesse est : nam quae ante acta sunt, sub vesperum acta finguntur. [...] Quod ego ex arte fieri non puto. Continuari nanque rei actum in Comcediis oportet ». Je traduis : «Il faut qu'on dorme dans cette comédie : en effet, les événements qui ont eu lieu sont censés avoir eu lieu le soir. [...] Je ne pense pas que cela soit fait dans les règles de l'art. Il faut en effet que l'action soit continue dans les comédies ».

11. En 1640, suite à une conversation animée avec son savant ami dans les jardins du Luxembourg, d'Aubignac fait paraître un Discours sur la troisième Comédie de Térence, intitulée Heautontimoroumenos: contre ceux qui pensent qu'elle n'est pas dans les règles anciennes du Poème Dramatique, Paris, Vve Camusat, 1640 (Bnf [YC.4483]). Gilles Ménage réplique aussitôt par la Réponse au discours sur la comédie de Térence, intitulée Heautontimoroumenos, où par occasion sont traitées plusieurs questions touchant le poème dramatique (Paris, Vve Camusat, 1640). Alors que le calme semble revenu, c'est Ménage qui souffle sur les braises en réimprimant cette Réponse dans l'ouvrage de ses mélanges, Aegidii Menagii Miscellanea, qu'il fait paraître en 1652 chez Courbé (cette réponse est l'avant-dernière pièce de ces mélanges, et est paginée de la page 25 à la page 102). Il fait précéder son propre texte par le Discours de l'abbé d'Aubignac, mais sans en indiquer l'auteur, et donc sans même citer celui qui est devenu son ennemi. Celui-ci réagit avec force et publie en 1656 l'ouvrage intitulé Térence justifié ou deux Dissertations concernant l'art du théâtre, dont la première est un Discours sur la troisième comédie de Térence, intitulée Heautontimoroumenos, pour montrer qu'elle est dans les règles des anciens Poètes, Et la seconde est une apologie de ce même discours, où sont traitées plusieurs maximes du poème dramatique. Avec diverses Questions curieuses et utiles à ceux qui recherchent la connaissance de l'Antiquité, Paris, G. de Luyne, 1656, titre qui indique clairement la posture critique de celui qui est sur le point de faire paraitre son chef-d'œuvre théorique, $L a$ Pratique du théâtre, édité l'année suivante, dans lequel il s'agit de fonder les règles en raison tout en s'appuyant sur le modèle légué par les Anciens : justifier Térence, c'est légitimer la régularité à la fois par l'autorité et par le bon sens.

12. Après l'édition de Daniel Heinsius en 1618 à Amsterdam et celle, bilingue, de Michel de Marolles en 1659 en France, c'est l'édition de Madame Dacier en 1688 qui fait autorité à la fin du XVII ${ }^{\mathrm{e}}$ siècle.

13. Ménage, Discours de Ménage sur l'Heautontimoroumenos de Térence, Utrecht, Antoine Schouten, 1690. Nous l'abrègerons ici en Discours.

14. Ibid., p. 218.

15. Ibid., p. 171-195.

16. Ibid., p. 211.

17. Ibid., p. 142 : «Il est constant d'ailleurs que notre comédie commence lorsque le soleil se couche, comme il paraît par le mot de vesperacit à la $3^{\mathrm{e}}$ scène du second acte ».

18. Histoire de la littérature française [1948], Paris, Albin Michel, vol. 1, 1997, p. 278.

19. M. Descotes, Histoire de la critique dramatique en France, Tübingen-Paris, Gunter Narr-JeanMichel Place, 1980, p. 27.

20. E. Samfiresco, Ménage polémiste, philologue, poète, Genève, Slatkine, 1971, p. 112.

21. Cité par P. Pasquier, op. cit., p. 107.

22. Térence justifié, op. cit., p. 4.

23. Discours, op. cit., p. 139.

24. Expression de E. G. Lessing dans La Dramaturgie de Hambourg, trad. Edouard de Suckau, Paris, Didier, 1896, p. 224-225.

25. Poetices libri septem, Lyon, Antonin Vincent, 1561, VI ("Qui est hypercriticus»), chap. 3 (éd. L. Deitz et Gregor Vogt-Spira, Stuttgart-Bad Cannstatt, Fromman-Holzboog, 1994-2011, vol. 5, p. 54-56). La traduction est de D. Voisin, que je remercie. 
26. H. W. Lawton, "L'Heautontimoroumenos de Térence. J.-C. Scaliger et les unités dramatiques", dans Mélanges d'histoire littéraire de la Renaissance offerts à Henri Chamard, Paris, Nizet, 1951, p. 205-209.

27. Les comédies de Térence traduites en français par Madame Dacier [Barbin, 1688]. Je la cite dans l'édition de 1695 parue à Lyon, chez Horace Molin, vol. II, p. 223.

28. «On trouverait aujourd'hui des occasions pour faire avec grâce ce qu'ils [Ménandre et Térence] ont fait », ibid., p. 225.

29. Ibid., p. 224.

30. Ces phrases de Beaumarchais se situent entre les deux premiers actes d'Eugénie [1767], juste après la description du premier «jeu d'entracte » (Euvres, éd. P. Larthomas, Paris, Gallimard, «Bibliothèque de la Pléiade », 1988, p. 154).

31. Térence justifié, op. cit., p. 18.

32. Ibid., p. 20.

33. Loc. cit.

34. Discours, op. cit., p. 257.

35. Ibid., p. 263.

36. Ibid., p. 263-264.

37. E. G. Lessing, Dramaturgie de Hambourg, op. cit., p. 224-225.

38. Discours sur la poésie dramatique, édité avec Le Père de famille [1758], dans Diderot, CEuvres esthétiques, éd. P. Vernière, Paris, Garnier Frères, « Classiques Garnier », 1959, p. 243.

39. Térence justifié, op. cit., p. 17.

40. H. C. Lancaster, A History of French Dramatic Literature in the XVII ${ }^{\text {th }}$ Century, Baltimore, Maryland, The Johns Hopkins Press, 1929 ; Paris, PUF, 1929-1942 (9 vol.).

41. Dictionnaire analytique des cuvres théâtrales françaises $d u X{ }^{e} I^{e}$ siècle, Paris, Honoré Champion, 1998. Nous l'abrègerons ici en Dictionnaire.

42. Voir par exemple S. Guellouz, Le théâtre au XVII siècle, Rosny, Bréal, 2002, p. 58 : « l'intrigue ne peut en aucune manière tenir en vingt-quatre heures ».

43. On lit aussi "la nuit est certainement tombée", dans l'édition critique de Cassandre de Boisrobert, disponible en ligne sur le site de la Bibliothèque dramatique du CELLF : http:// bibdramatique.paris-sorbonne.fr/boisrobert_cassandre/front-2\#front-2-6.

44. "L'unité de temps est respectée », passim.

45. H. C. Lancaster, op. cit., part II, v. 1, p. 190 («Les unités de temps et de lieu sont respectées »).

46. Ibid., part II, v. 1, p. 192 ( Le temps ne dépasse pas vingt-quatre heures »). On trouve aussi souvent l'expression « the time is about twenty-four hours» (« la temporalité est d'environ vingtquatre heures »).

47. Ibid., part II, v. 1, p. 183 (« The play is composed in accordance with the classical system : twenty-four hours »).

48. Dictionnaire, op. cit., p. 209.

49. H. C. Lancaster, op. cit., part III, v. 2, p. 442 (" the rapid action takes place in a few hours »).

50. Dictionnaire, op. cit., p. 176.

51. Ibid., p. 58.

52. Térence justifié, op. cit., p. 2.

53. « Rodrigue a pris haleine en vous la racontant» déclare Don Diègue au roi Don Fernand (Le Cid, v. 1457).

54. Corneille, Discours de la tragédie, dans E Euvres complètes, éd.G.Couton, Paris, Gallimard, «Bibliothèque de la Pléiade », 1987, vol. 3, p. 171-172.

55. Corneille, Examen du Cid, dans Euvres complètes, op. cit., vol. 1 [1980], p. 704.

56. La Pratique du théâtre, I, vII, « De l'étendue de l'action théâtrale ou du Temps et de la durée convenables au Poème Dramatique. », op. cit., p. 183.

57. Discours, op. cit., p. 239. 
58. «Sabine entre angoissée par un songe qu'elle a fait, au moment de la sieste selon toute vraisemblance ", Dictionnaire, op. cit., p. 561.

59. Entre 1635 et 1651, cette question ne se pose guère et l'on peut citer ainsi La Mort de Brute et Porcie de Guérin de Bouscal, Didon de Scudéry, Lucrèce de Du Ryer, La Lucresse romaine de Chevreau, La Mort de Pompée de Chaulmer, Antigone de Rotrou, Cammane de La Caze, Saül de Du Ryer, Alinde de La Mesnardière, Agamemnon d'Arnaud.

60. Pièces signées respectivement par Pierre Corneille, Jean Mairet, Jean Rotrou et Paul Scarron.

61. Bruxelles, 1669.

\section{AUTEUR}

\section{HÉLÈNE BABY}

Université Côte d'Azur-CTEL 